\title{
CONCENTRACIÓN SEMANAL DE AEROPOLEN EN LA CIUDAD DE PALENCIA, 1990-92.
}

\author{
Baudilio HERRERO
}

\begin{abstract}
RESUMEN. Concentración semanal de aeropolen en la ciudad de Palencia, 1990-92. En este trabajo se presentan los datos del contenido polínico de la atmósfera de la ciudad de Palencia, registrado a lo largo de tres años consecutivos de muestreo (1990-92), empleándose un captador volumétrico de filtracción activa, tipo CAP2. Durante este período se identificaron 88 tipos polínicos diferentes, de los cuales, 27 tuvieron porcentajes de representación superior al $0,15 \%$ respecto al polen total que anualmente se recogió, estos tipos conforman el denominado espectro polínico principal de esta estación de muestreo. El polen procedente de especies herbáceas (Poaceae, Chenopodiaceae, Plantago, Urtica, etc.) con un 53,79\% fue el predominante, el polen arbóreo (Quercus, Populus, Cupressaceae, etc.) supone un 42,11\%, y el polen procedente de especies arbustivas (Ericaceae, Sambucus, etc.) sólo representó el 4,10\%. El período de máxima emisión polínica se concentra en los meses de mayo y junio, debido a las aportaciones de polen procedente de Poaceae y Quercus, que en conjunto suman el 47,25\% del polen recogido durante el trienio muestreado. El polen de origen herbáceo aparece durante todo el año, y sus máximas concentraciones se registran en primavera, coincidiendo con los máximos niveles de polen de origen arbóreo en la atmosfera.
\end{abstract}

Palabras clave. Aerobiología, polen, filtración activa, Palencia.

ABSTRACT. Weekly aeropollen concentration in Palencia city, 1990-92. The aim of this work is to present the data of the pollen concentrations in the atmosphere of Palencia city. It was recorded over three consecutive years of sampling (1990-92). A volumetric pollen trap of active filtration, type CAP2, was used. During this time, 88 different pollen types were identified, 27 occured at more than $0,15 \%$ of the total pollen recorded annually. These types formed the main pollen spectrum of this sampling station. Pollen coming from herbaceous species (Poaceae, Chenopodiaceae, Plantago, Urtica, etc.) was predominant (53,79\%); arboreal pollen (Quercus, Populus, Cupressaceae, etc.) represented 42,11\%, and pollen coming from shrub species (Ericaceae, Sambucus, etc.) only represented 4,10\%. May and June was the time of the year, with the maximum pollen emission concentrated in the air. This was due to the quantities of pollen coming from Poaceae and Quercus, which together represented 47,25\% of the pollen recorded over the three-year sample. Herbaceous pollen appeared throughout the year, with maximum concentrations recorded in spring, and coinciding with the maximum levels of arboreal pollen in the atmosphere.

Key words. Aerobiology, pollen, active filtration, Palencia, Spain.

\section{INTRODUCCIÓN}

El conocimiento del contenido polínico presente en la atmósfera de un área geográfica determinada, ofrece posibilidades de aplicación en diferentes campos del conocimiento, ligados a la agricultura (Cour \& Van Campo, 1980), medio ambiente (Doskey \& Uroagwu, 1992) y a la medicina (Subiza Martín et al., 1988), este hecho ha provocado a nivel mundial, un 
incremento del interés por conocer el contenido de polen en la atmósfera de diferentes ciudades.

El objetivo de este estudio fue la obtención de datos cualitativos y cuantitativos del polen que aparece en la atmósfera de la ciudad de Palencia. Este trabajo amplia los estudios parciales llevados a cabo por los autores (González Minero et al., 1993; Herrero, 1995), y complementa los trabajos realizados en otras ciudades de Castilla y León (Linares et al., 1983; Fernández González et al., 1993; Fernández González \& Valencia Barrera, 1995). En el presente trabajo, se expone un análisis de los tipos polínicos más importantes, aportándose el tipo de período de polinización, el momento de máxima concentración y la evolución de sus concentraciones medias semanales, comparándolo con otras ciudades y aportando datos de su variabilidad a lo largo del período muestreado. Asímismo se destacan los tipos polínicos que mayor incidencia pueden tener en las polinosis del área estudiada. A partir de 1992, el muestreo se ha interrumpido.
Palencia se encuentra a $42^{\circ} 0^{\prime}$ de latitud norte y $4^{\circ} 32^{\prime}$ de longitud oeste, a una altura media de $743 \mathrm{~m}$ sobre el nivel del mar, situada en la zona sur de la provincia, en el valle del río Carrión y entre dos unidades morfoestructurales: Tierra de Campos y los páramos calcáreos. Aparece enclavada en la cuenca del Duero, dentro del cuadrante noroeste de la Península Ibérica.

La temperatura media anual alcanza los $11,7^{\circ} \mathrm{C}$, presentando largos inviernos y cortos veranos de contrastes térmicos muy acusados. Las precipitaciones anuales son bajas, $351 \mathrm{~mm}$ de promedio anual, distribuidas de forma bastante homogénea entre las estaciones de primavera, otoño e invierno. Los vientos predominantes son de componente suroeste, penetrando por la cuenca del Duero.

A lo largo del trienio muestreado, la temperatura media fue de $10,6{ }^{\circ} \mathrm{C}$, la precipitación media anual alcanzó los $315 \mathrm{~mm}$ (fig. 1), y los vientos predominates de componente oeste.

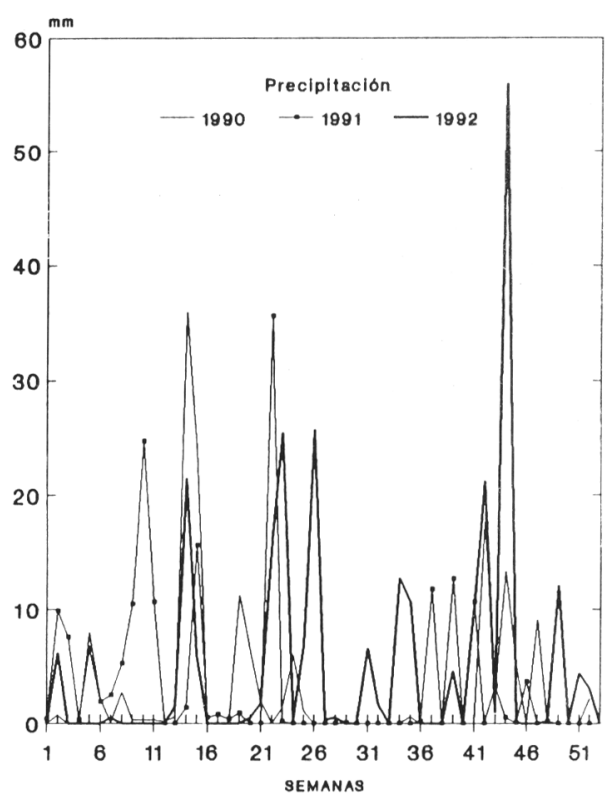

Figura 1. Datos meteorológicos de Palencia, en el período 1990-92. Meteorological data for Palencia, between 1990 and 92 . 
La vegetación potencial pertenece biogeograficamente al piso supramediterráneo de la Región Mediterránea, provincia Castellano-Maestrazgo-Manchega, sector Castellano-Duriense (Rivas Martínez, 1987), estando constituido mayoritariamente por series de quejigares (Quercus faginea) y encinares (Quercus rotundifolia), que ocupan aproximadamente un $10 \%$ de la superficie del entorno de la ciudad de Palencia, el $90 \%$ del área circundante corresponde a vegetación agrícola de cultivos extensivos cerealistas, recientemente la superficie destinada a cultivos de remolacha y alfalfa se ha incrementado notablemente.

\section{MATERIAL Y MÉTODOS}

Para llevar a cabo este estudio se empleó un captador de filtración activa CAP2 (SuárezCervera \& Seoane-Camba, 1983), situado a 12 $m$ de altura, en la azotea del edificio que alberga la Escuela Técnica Superior de Ingenierías Agrarias, este centro se ubica en la zona suroccidental de la ciudad. Las muestras se recogieron diariamente sobre un filtro de ester de celulosa de $5 \mu \mathrm{m}$ de poro y $70 \mathrm{~mm}$ de diámetro, después de estar expuesto durante 24 horas en el captador y habiéndose filtrado 4 $\mathrm{m}^{3}$ de aire.

Los filtros para su análisis a microscopía óptica, se colocan sobre portaobjetos impregnados en aceite de inmersión y cubiertos por sus correspondientes cubreobjetos, obteniéndose 3 preparaciones por cada día muestreado. Se llevó a cabo un análisis completo del filtro, y los resultados se expresaron como el número de pólenes por $\mathrm{m}^{3}$. Se contabilizaron e identificaron 88 tipos, de ellos se calcularon sus frecuencias relativas y se seleccionaron los 27 tipos polínicos más abundantes. Asímismo se cuantificó el total de pólenes recogidos diariamente.

El estudio se ha llevado a cabo durante 3 años consecutivos, desde enero de 1990 hasta diciembre de 1992.

Los 27 tipos de pólenes más importantes en el espectro de Palencia, fueron objeto de seguimiento cuantitativo más amplio. A partir de las concentraciones diarias se calcularon las concentraciones medias semanales, con el fin de facilitar la interpretación de tendencias anuales. Los tipos polínicos establecidos coinciden mayoritariamente con los descritos por Valdés et al., (1987), considerándose los límites taxonómicos elásticos, así pueden incluirse en un tipo: pólenes de una sola especie, de un género y hasta de dos familias.

Para los tipos polínicos más importantes se especifica el porcentaje de representación anual, el período polínico principal (PPP) según los criterios de Nilsson \& Persson (1981), y la semana de máxima concentración polínica (SMCP).

Asimismo, se han elaborado figuras en las cuales se representan las concentraciones medias semanales del trienio muestreado, considerando el polen total, pólenes procedentes de especies arbóreas, arbustivas y herbáceas, asi como los 12 tipos polínicos con mayor representación en el espectro de Palencia, los cuales aparecen ordenados en sentido decreciente de porcentaje de representación.

\section{RESULTADOS Y DISCUSIÓN}

A lo largo de los 3 años muestreados, se han encontrado 88 tipos polínicos en la atmósfera de Palencia, de los cuales 27 tuvieron una representación superior al $0,15 \%$ del polen total, dichos tipos se exponen en la tabla I ordenados alfabeticamente por las familias a las que pertenecen, junto con los valores de las agrupaciones polínicas, considerando el porte morfológico de la planta emisora. La vegetación presente en el área de estudio se ve reflejada en el contenido polínico de la ciudad, los tipos 


\begin{tabular}{|c|c|c|c|c|c|}
\hline \multirow[t]{2}{*}{ Tipo polínico } & \multicolumn{3}{|c|}{ Porcentaje } & \multirow[t]{2}{*}{ PPP } & \multirow[t]{2}{*}{ SMCF } \\
\hline & 1990 & 1991 & 1992 & & \\
\hline Acer negundo & 0,31 & 0,57 & 0,11 & $8-20$ & 12 \\
\hline Artemisia vulgaris & 1,61 & 0,18 & 0,37 & $20-38$ & 29 \\
\hline Helianthus annuus & 0,16 & 0,34 & 0,46 & $28-34$ & 28 \\
\hline Alnus glutinosa & 2,85 & 0,64 & 2,61 & $2-15$ & 10 \\
\hline Betula pubescens & 1,61 & 0,18 & 2,4 & $4-23$ & 16 \\
\hline Corylus avellana & 0,55 & 0,46 & 0,72 & $47-18$ & 7 \\
\hline Brassicaceae & 3,31 & 5,23 & 2,79 & $|2-3|$ & 20 \\
\hline Sambucus nigra & 0,72 & 0,52 & 0,63 & $17-22$ & 21 \\
\hline Chenopodiaceae & 6,14 & 4,77 & 8,71 & $24-40$ & 36 \\
\hline Cupressaceae & 1,72 & 7,92 & 4,89 & $4-17$ & 7 \\
\hline Carex & 0,77 & 0,67 & 1,02 & $6-37$ & 29 \\
\hline Ericaceae & 0,85 & 1,19 & 0,74 & $4-41$ & 20 \\
\hline Mercurialis tomentosa & 0,75 & 0,86 & 0,84 & $14-38$ & 20 \\
\hline Ononis repens & 1,03 & 0,85 & 0,65 & $20-36$ & 24 \\
\hline Quercus & 19,96 & 21,26 & 14,73 & $17-25$ & 23 \\
\hline Fraxinus angustifolia & 2,74 & 0,18 & 0,46 & $2-13$ & 12 \\
\hline Ligustrum lucidum & 0,41 & 0,26 & 0,32 & $20-35$ & 32 \\
\hline Pinus & 2,48 & 4,77 & 6,01 & $9-38$ & 20 \\
\hline Plantago & 4,46 & 2,95 & 5,01 & $12-37$ & 18 \\
\hline Platanus hybrida & 0,27 & 0,23 & 0,53 & $11-18$ & 12 \\
\hline Poaceae & 32,85 & 22,94 & 25,58 & $5-47$ & 21 \\
\hline Polygonum & 0,18 & 0,24 & 0,3 & $23-35$ & 24 \\
\hline Rumex & 1,51 & 2,2 & 3,58 & $9-45$ & 20 \\
\hline Populus alba & 5,24 & 8,73 & 6,7 & $10-16$ & 12 \\
\hline Salix & 1,72 & 1,06 & 2,45 & $15-23$ & 17 \\
\hline Ulmus minor & 0,92 & 1,08 & 0,67 & $8-20$ & 12 \\
\hline Urtica & 1,44 & 7,12 & 3,95 & $3-44$ & 13 \\
\hline Otros tipos polínicos & 3,98 & 3,49 & 2,77 & & \\
\hline Polen arbóreo & 38,51 & 46,1 & 41,79 & $10-26$ & 24 \\
\hline Polen arbustivo & 4,45 & 4,12 & 3,74 & $14-46$ & 22 \\
\hline Polen herbáceo & 56,74 & 49,78 & 54,45 & $7-44$ & 23 \\
\hline Polen total & 100 & 100 & 100 & $5-45$ & 21 \\
\hline
\end{tabular}

Tabla 1. Espectro polínico principal de Palencia y grupos polínicos morfológicos, indicándose los porcentajes anuales alcanzados, el período de polinización principal (PPP), y la semana de máxima concentración polínica (SMCP), durante el trienio 1990-92. The principal pollen spectrum of Palencia and morphologic pollen types. It shows the percentage of appearence, the principal pollen period (PPP), and the week maximum pollen concentration (WMPC), during the three-year sample (1990.92).

más abundantes son: Poaceae $(28,80 \%)$, Quercus $(19,45 \%)$, Populus $(6,78 \%)$, Chenopodiaceae-Amaranthaceae $(6,20 \%)$ y Pinus (4,04\%). Los tipos polínicos menos representados en la atmósfera de la ciudad de Palencia, con porcentajes inferiores a $0,40 \%$, pero incluidos dentro del espectro polínico principal, fueron: Acer negundo (0,36\%), Ligustrum lucidum $(0,34 \%)$, Platanus hybrida (0,31\%), Helianthus annuus $(0,29 \%)$ y Polygonum aviculare $(0,23 \%)$.
En base al período polínico principal de los diferentes tipos polínicos estudiados (tab. 1), se pueden establecer 3 grupos:

- Pólenes con período polínico principal corto, inferior a 10 semanas, se trata de tipos polínicos que incluyen pocas especies y presentan una floración corta, en este grupo se engloban: Helianthus annuus, Sambucus nigra, Quercus, Platanus hybrida, Populus alba y Salix.

- Los tipos con un período polínico principal de tipo medio, que comprende entre 10 y 15 

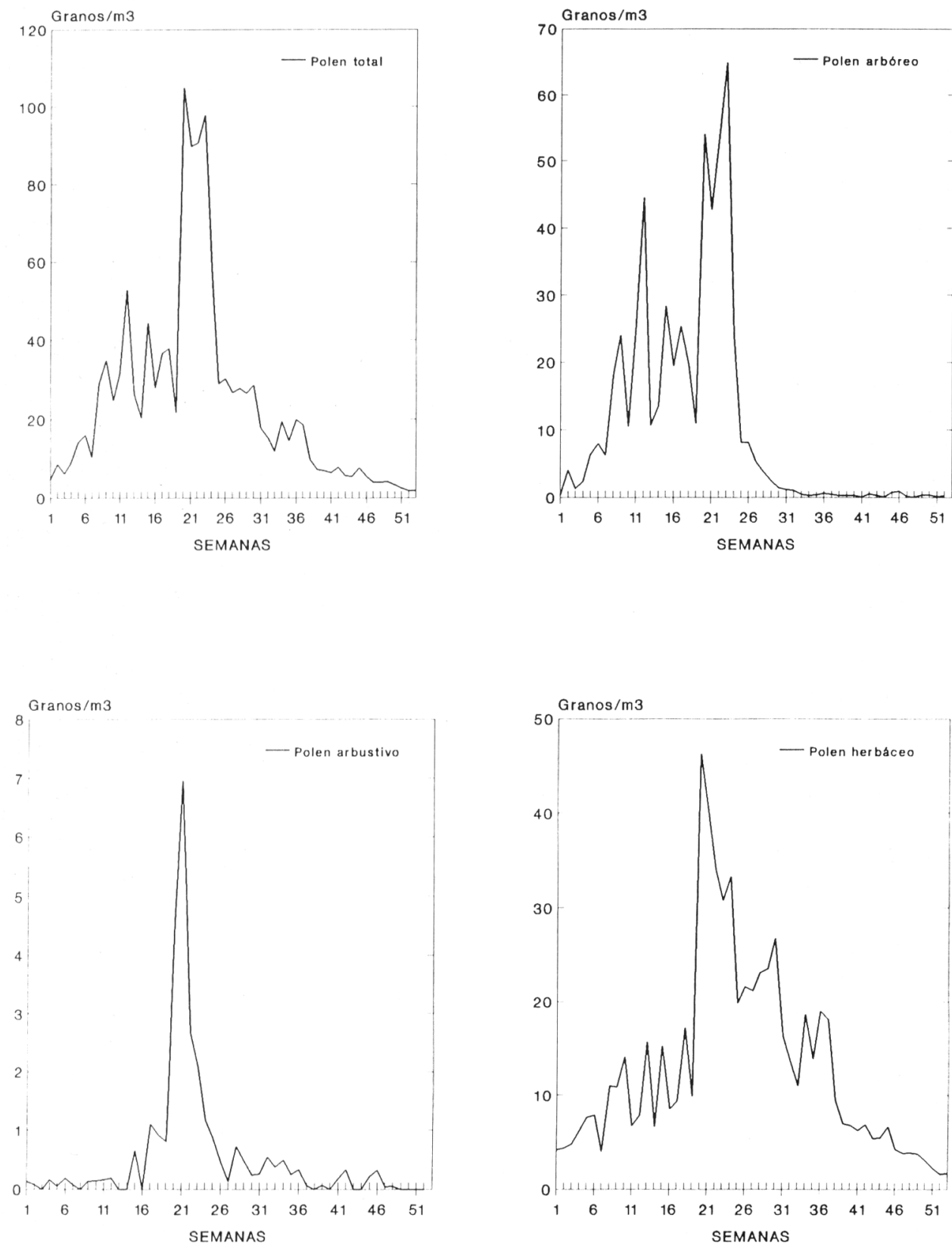

Figura 2. Variación de la concentración media semanal del polen total y de los pólenes procedentes de especies de porte arbóreo, arbustivo y herbáceo, en el período 1990-92. Variation of weekly mean pollen concentrations of total pollen and of arboreal, shrub and herbaceous pollen, between 1990 and 92 . 
semanas, se corresponde con: Acer negundo, Alnus glutinosa, Cupressaceae, Fraxinus angustifolia, Polygonum aviculare y Ulmus minor.

- Tipos con período de polinización largo, superior a 15 semanas: Artemisia vulgaris, Betula pubescens, Corylus avellana, Brassicaceae, Chenopodiaceae-Amaranthaceae, Carex, Ericaceae, Mercurialis tomentosa, Ononis repens, Ligustrum lucidum, Pinus, Plantago, Poaceae, Rumex y Urtica. Sus registros en la atmósfera, presentan oscilaciones anuales debido a que estos tipos polínicos en ocasiones se corresponden con una familia o varios géneros integrados por numerosos taxones con floraciones graduales, o bien a que son tipos que emiten polen de forma gradual e intermitente en pequeñas cantidades.

En la ciudad de Palencia, el polen aparece mayoritariamente durante los meses de marzo a julio (fig. 2), los valores máximos se registran hacia la semana 21 , mediados de mayo, duplicándose respecto al período precedente, estas altas concentraciones se deben mayoritariamente a polen de especies arbóreas, coincidiendo con altas emisiones a la atmósfera de polen de Quercus. La curva de presencia se haya desplazada hacia finales de primavera, frente a los espectros de ciudades más meridionales de la Península Ibérica, como Sevilla (González Romano, 1992) que presenta altas concentraciones polínicas desde principios de febrero.

El polen arbóreo presenta dos picos de máximas concentraciones medias semanales, uno en marzo debido al polen de Populus y Cupressaceae $(11,40 \%)$, y otro en mayo reflejo de la polinización de Quercus $(19,45 \%)$. El polen procedente de especies de porte arbustivo, presenta un porcentaje del $4,10 \%$ respecto al polen total recogido durante el trienio estudiado, coincidiendo con los porcentajes señalados en ciudades como, Barcelona (Belmonte, 1988), la concentración máxima registrada a finales de mayo, se corresponde con floraciones de Salix, Sambucus nigra y Ligustrum lucidum, su registro continuo a lo largo del año, se debe al polen de Ericaceae. El polen herbáceo aparece durante todo el año, con un máximo entre mayo y agosto, su porcentaje de representación supera en un $10 \%$ al polen de origen arbóreo. Las variaciones entre el polen de origen arbóreo y polen herbáceo (tab. 1), puede explicarse por las variaciones cíclicas en la producción de polen por parte de las especies arbóreas (Emberlin et al., 1990), que hace que se eleve el porcentaje de polen arbóreo y descienda la frecuencia del polen herbáceo.

En cuanto a los tipos que forman parte del espectro polínico principal de la ciudad de Palencia (figs. 3 y 4), destacan Poaceae con un porcentaje del $28,80 \%$, resultado de su caracter anemófilo, la amplitud en el número de especies que engloba y la variabilidad de medios que ocupa; aparece en la atmósfera durante todo el año, alcanzando sus máximas concentraciones entre los meses de abril y julio. Las concentraciones encontradas son similares a las observadas en otras ciudades próximas, como León (Fernández González et al., 1993). Los picos máximos de polen de poáceas en el aire, coinciden con épocas de labores agrícolas relacionadas con manipulación de los cereales. El porcentaje de representación en el año 1990 fue notablemente superior a los otros dos años, oscilaciones que también han sido señaladas en otros estudios (Buck \& Levetin, 1985), fruto de la heterogeneidad de especies y hábitats. Asímismo, la lluvia caida en 1990 fue superior a los registros de 1991 y 1992, lo cual ha podido favorecer una mayor presencia de poáceas, dado que se trata mayoritariamente de especies anuales, las cuales son influenciadas en gran medida por lluvias preestacionales.

El tipo polínico Quercus, con un porcentaje de representación del 19,45\%, aparece en la atmósfera durante los meses de 

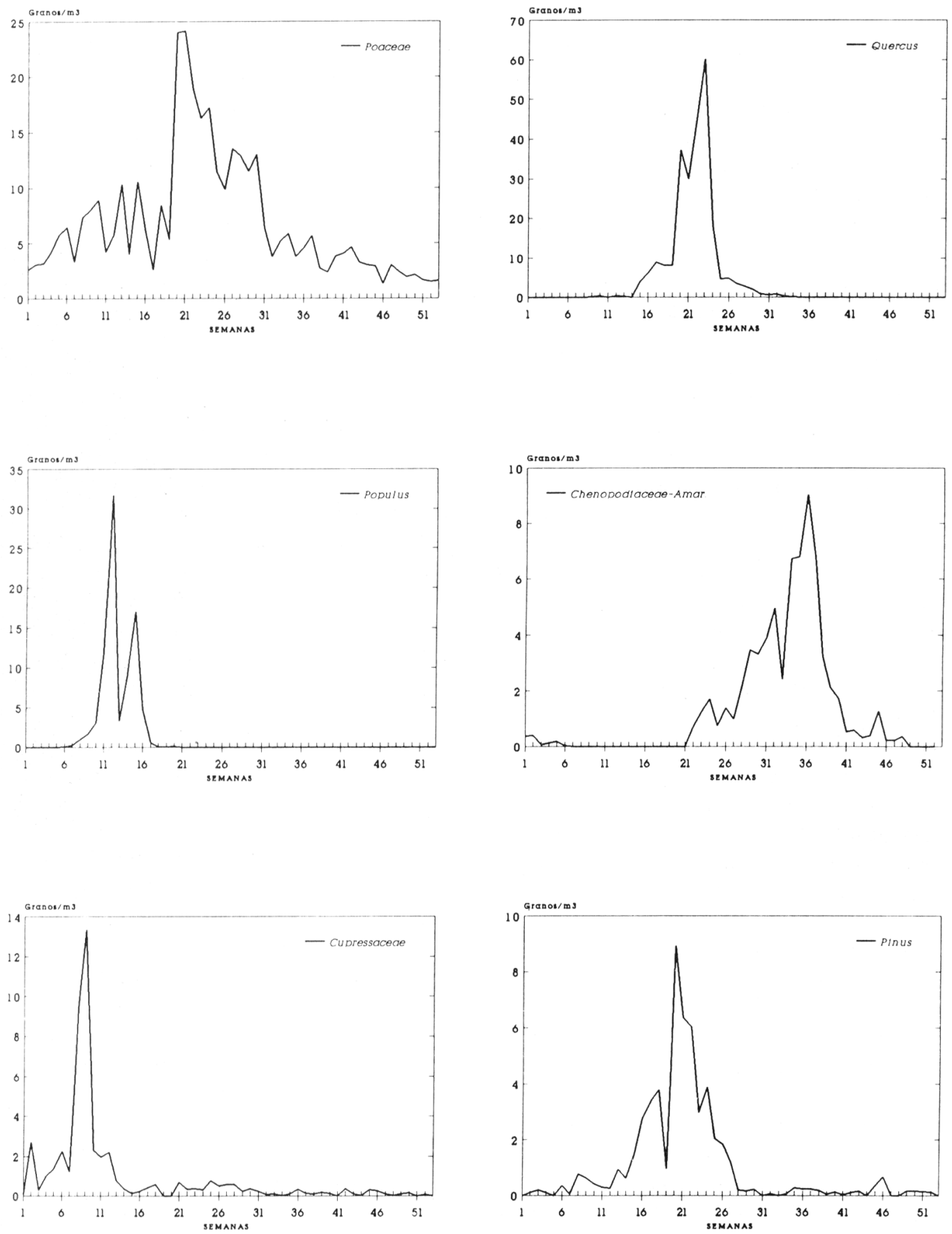

Figura 3. Variación de la concentración media semanal del polen de los tipos: Poaceae, Quercus, Populus, Chenopodiaceae, Cupressaceae y Pinus, en el período 1990-92. Variation of weekly mean pollen concentrations of the following types: Poaceae, Quercus, Populus, Chenopodiaceae, Cupressaceae and pinus, between 1990 and 92. 
abril y julio, sus concentraciones máximas se registran a mediados de junio, el período de presencia se prolonga debido a la existencia de 2 especies de Quercus en el área ( $Q$. faginea y $Q$. rotundifolia), con períodos de floración consecutivos.

Populus alba tiene su floración entre los meses de febrero a abril, sus concentraciones máximas se presentan en la segunda quincena de marzo, y su importancia contrasta con la ausencia de este tipo de polen en los espectros de ciudades como Sevilla (González Romano et al., 1992), y Huelva (González Minero, 1993); le sigue en importancia el tipo polínico Chenopodiaceae-Amaranthaceae, su presencia se extiende practicamente durante todo el año, sus floraciones máximas tienen lugar a primeros del mes de septiembre, los niveles polínicos son inferiores a los registrados en áreas más meridionales de la Península, como Almeria (Belmonte \& Roure, 1991).

El tipo Cupressaceae engloba principalmente pólenes de los géneros Chamaecyparis, Cupressus, Juniperus y Thuja; debido a esta heterogeneidad, presenta una curva de incidencia que va desde diciembre a abril, sus concentraciones máximas se situan a mediados de febrero. Este tipo polínico tiene gran importancia en los espectros polínicos de ciudades como Córdoba, Granada y Málaga (Domínguez et al., 1995; Díaz de la Guardia, 1995; Cabezudo et al., 1995), donde constituye el tipo polínico más importante. En cuidades como Teruel (Belmonte et al., 1995), alcanza un porcentaje superior al $40 \%$. En todas estas ciudades se race presente en la atmósfera durante el invierno, en contraste con regiones como Bursa, Turquia (Bicakci et al., 1996) que está presente en la atmósfera durante la primavera. A pesar de que su caracter alergógeno ha sido citado por diversos autores, en la zona de estudio no origina graves problemas alérgicos.

El tipo Pinus alcanza un porcentaje muy inferior al encontrado en ciudades nórdicas
(Kotzamanidou \& Nilsson, 1977), se hace presente practicamente durante todo el año, debido a fenómenos de reflotación, alcanzando sus máximas concentraciones a mediados de mayo.

Las concentraciones de otros tipos polínicos encontrados (fig. 4) son: Plantago (4,06\%), Urtica $(3,98 \%)$, Brassicaceae $(3,88 \%)$, Rumex $(2,19 \%)$, Alnus glutinosa $(2,02 \%)$ y Salix $(1,64 \%)$.

En el mes de mayo aparecen las máximas concentraciones de los pólenes de los tipos: Plantago, Brassicaceae, Rumex y Salix.

La presencia prolongada de los tipos polínicos: Plantago, Urtica y Rumex, aun alcanzando bajas concentraciones, determina el que tengan gran trascendencia como causantes de polinosis en Palencia, hecho que coincide con los resultados descritos para la ciudad de Valladolid por Linares López et al., (1983). El polen de Alnus glutinosa se registra en el primer trimestre del año, alcanzando su máxima concentración a primeros de marzo, su baja concentración en 1991 (tab. 1), puede ser explicada por la ritmicidad bianual atribuida a este polen por Troise et al., (1992). Las brasicáceas, dada su facilidad para colonizar medios antrópicos, desarrollan abundantes poblaciones productoras de polen, que a pesar de tener mayoritariamente polinización entomófila, también representan un importante tipo polínico de dispersión anemófila.

Los otros 15 tipos que constituyen el espectro polínico principal, poseen porcentajes de representación inferiores al $1,50 \%$, encontrándose que sus emisiones polínica máximas se corresponde con tres períodos del año (tab. 1):

- A finales de invierno aparecen las polinizaciones máximas en: Acer negundo, Corylus avellana, Fraxinus angustifolia, Platanus hybrida y Ulmus minor.

- En la primavera, desde finales de abril y en el mes de mayo, se contabilizaron las concentraciones más elevadas en: Betula pubescens, Sambucus nigra, Ericaceae, 

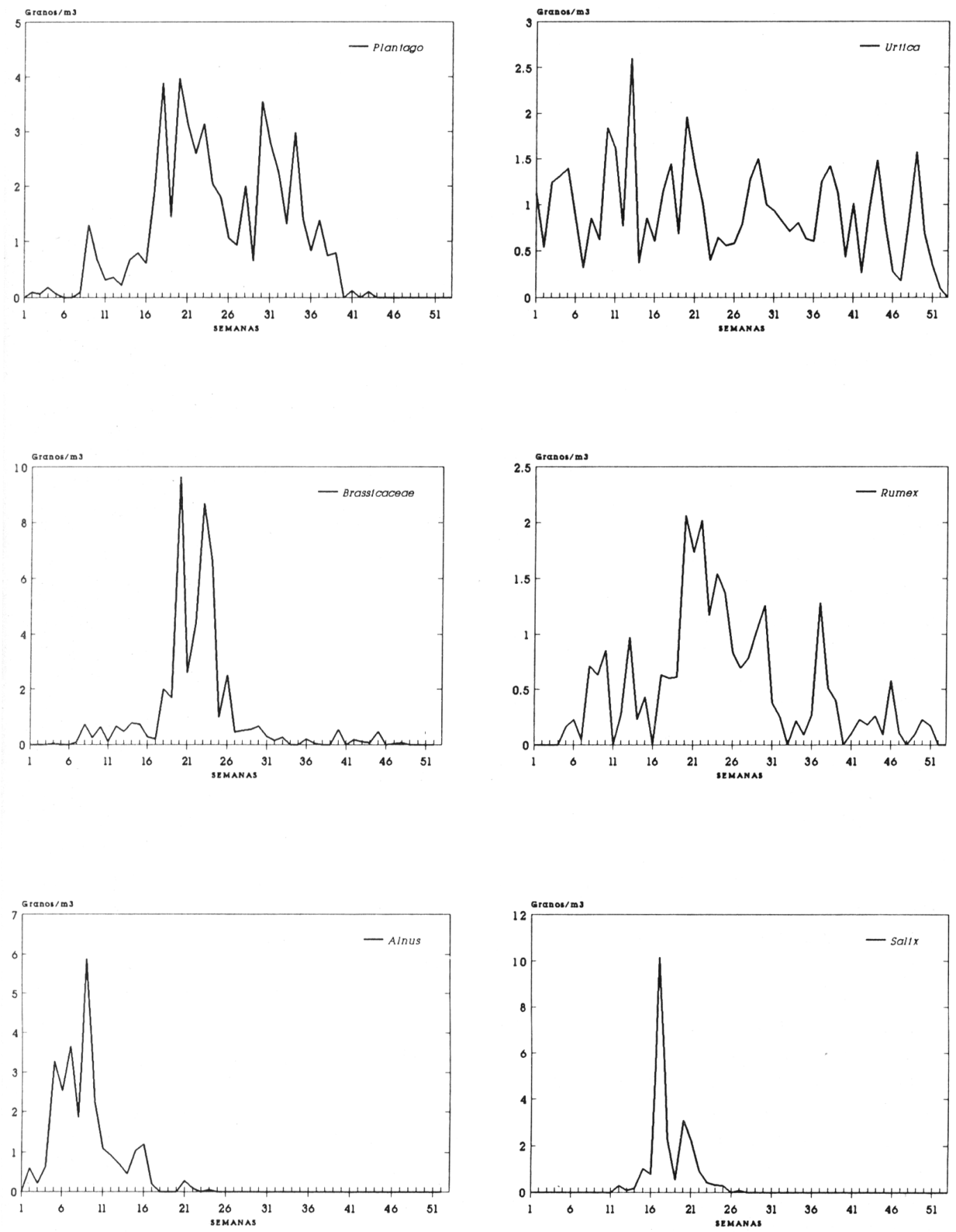

Figura 4. Variación de la concentración media semanal del polen de los tipos: Plantago, Urtica, Brassicaceae, Rumex, Alnus y Salix, en el período 1990-92. Variation of weekly mean pollen concentrations of the following types: Plantago, Urtica, Brassicaceae, Rumex, Alnus and Salix, between 1990 and 92. 
Mercurialis tomentosa, Ononis repens y Polygonum aviculare.

- Durante el verano se registran los picos máximos en la concentración de: Artemisia vulgaris, Helianthus annuus, Carex y Ligustrum lucidum.

\section{BIBLIOGRAFÍA}

BELMONTE, J. -1988- Concentración polínica en la atmósfera de Barcelona. Orsis 3:67-75.

BELMONTE, J. \& J.M. ROURE -1991Characteristics of the aeropollen dynamics at several localities in Spain. Grana 30:9-15.

BELMONTE, J., J.M. ROURE \& M. LABORDA 1995- Aerobiología de Aragón. Teruel. REA 1:61-64.

BICAKCI, A., O. INCEOGLU, N. SAPAN \& H. MALYER -1996- Airborne pollen calendar of the central region of Bursa (Turkey). Aerobiologia 12(1):43-46.

BUCK, P. \& E. LEVETIN -1985-Airborne pollen and mola spores in a subalpine environment. Annal of Allergy 55(6):794-801.

CABEZUDO, B., M.M. TRIGO y M. RECIO - 1995 Aerobiología de la Costa del Sol. Málaga. REA 1:47-49.

COUR, P. \& M. VAN CAMPO -1980- Prévisions de récoltes à partir de l'analyse du contenu pollinique de l'atmosphère. C.R. Acad.Sc. Paris 290:1043-1046.

DÍAZ DE LA GUARDIA, C. -1995- Aerobiología de Andalucia Oriental. Granada. REA 1:43-45.

DOMÍNGUEZ, E., C. GALÁN y F. INFANTE 1995- Aerobiología de Andalucia Central. Córdoba. REA 1:39-42.

DOSKEY, P.V. \& B.J. UROAGWU -1992Macronutrient chemistry of pollen. Commun. Soil. Sci. Plant. Anal. 23(1 y 2):15-23.

EMBERLIN, J.C., J. NORRIS-HILL \& R.H. BRYANT -1990-A calendar for tree pollen in London. Grana 29:301-309.

FERNÁNDEZ GONZÁLEZ, D., M. SUÁREZCERVERA, T. DÍAZ GONZÁLEZ y R.M. VALENCIA BARRERA - 1993- Airborne pollen and spores of León (Spain). Int. J. Biometeorol. 37:89-95.

FERNÁNDEZ GONZÁLEZ, D. y R.M. VALENCIA BARRERA -1995- Red Española de aerobiología. Estación de la Universidad de León. REA 1: 81-85.
GONZÁLEZ MINERO, F.J. -1993-Calendario polínico de Huelva y su relación con la polinosis y agricultura. Tesis Doctoral. Universidad de Sevilla.

GONZÁLEZ MINERO, F.J., B. HERRERO y P. CANDAU -1993-Latitudinal study of allergenic pollen in two Spanish cities. J. Invest. Allergol. Clin. Immunol. 3(6):304-310.

GONZÁLEZ ROMANO, M.L., P. CANDAU y F.J. GONZÁLEZ MINERO - 1992- Pollen calendar of Seville and its relations to allergies. J. Invest. Allergol. Clin. Immunol. 2(6):232-238.

HERRERO, B. -1995- Estudio del contenido del polen y esporas en la atmósfera de la ciudad de Palencia. In: Tesis Doctorales 1994. Servicio de Publicaciones, Universidad de León, CD-ROM.

KOTZAMANIDOU, P. \& S. NILSSON -1977- On the pollen incidence and phenology of some trees in sourtern and central Sweden, 19741975. A preliminary study. Grana 16:195-198.

LINARES LÓPEZ, P.M., J.A. GÓMEZ CARRASCO y R. ANDIÓN DAPENA - 1983- Estudio aerobiológico mediante método volumétrico, de la atmósfera de Valladolid, durante los años 1981, 1982. Actas del IV Simposio de Palinología APLE. Barcelona. pp. 261-276.

NILSSON, S. \& S. PERSSON -1981- Three pollen spectra in the Stockholm region (Sweden), 19731980. Grana 20:179-182.

RIVAS MARTÍNEZ, S. -1987-Memoria del mapa de series de vegetación de España. ICONA. Madrid.

SUÁREZ-CERVERA, M. \& J.A. SEOANE-CAMBA 1983- Estudio del contenido polínico de la atmósfera de Barcelona según un nuevo método de filtración. Collectanea Botánica 14:587-615.

SUBIZA MARTÍN, E., F.J. SUBIZA GARRIDOLESTACHE y M. JEREZ LUNA -1988Polinosis (I). Palinología. Par. 70:11-55.

TROISE, C., S. VOlTOLINI, G. DELBONO \& A.C. NEGRINI -1992- Allergy to pollen from Betulaceae and Corylaceae in a Mediterranean area (Genoa, Italy). A ten-year retrospectyve study. J. Invest. Allergol. Clin. Immunol. 2(6):313-317.

VALDÉS, B., M.J. DÍEZ e I. FERNÁNDEZ - 1987 Atlas polínico de Andalucia Occidental. Instituto de Desarrollo Regional. Universidad de Sevilla. Excma. Diputación de Cádiz. Sevilla.

Aceptado para su publicación en Junio de 1997

Dirección del autor. Departamento de Ciencias Agroforestales, Universidad de Valladolid. Avenida Madrid, 57 34004-Palencia. 